\title{
VALORACIÓN DE NECESIDADES DEL PERSONAL DE FORMACIÓN, GESTIÓN Y ADMINISTRACIÓN DE LOS CENTROS ESPECIALES DE EMPLEO EN ASTURIAS
}

\author{
EVALUATION OF THE NEEDS OF STAFF IN SPECIAL CENTERS OF \\ EMPLOYMENT IN ASTURIAS REGION
}

\author{
Eva María Carrio* \\ Universidad de Oviedo
}

\section{RESUMEN}

Este trabajo es un análisis de necesidades que parte de la realidad a través de un estudio descriptivo de la misma.

Para evaluar las necesidades del personal de formación, gestión y administración que trabaja en los Centros Especiales de Empleo partimos de las propias percepciones de este personal sobre estos Centros, sobre la labor que desempeñan y sobre los problemas y dificultades a las que se enfrentan al tratar de favorecer la inserción laboral de las personas con discapacidad.

La información recogida se ha obtenido mediante la elaboración y aplicación de cuestionarios semiestructurados y entrevistas abiertas.

Palabras clave: Discapacidad, integración laboral.

\section{ABSTRACT}

This job is an analysis of needs that part of the reality through a descriptive study of the same one.

To evaluate the needs of the personnel of formation, management and administration that works in the Special Centers of Employment we leave from the own perceptions of this personnel upon these Centers, upon the work that they perform and upon the problems and difficulties to the ones that are faced al to try to favor the labor insertion of the persons with discapacidad.

* Licenciada en Psicopedagogía por la Universidad de Coruña. Posee la Suficiencia Investigadora otorgada por la Universidad de Oviedo en diciembre del 2000. Actualmente es Becaria de Investigación en el Departamento de CC. de la Educación de dicha Universidad y está realizando la tesis doctoral sobre los Centros Especiales de Empleo de Asturias. 
The information collected has been obtained by means of the elaboration and application of questionnaires semiestructurados and open interviews.

Key words: Disability, labour integration.

\section{Justificación de la investigación}

Los Centros Especiales de Empleo juegan un importante papel en los procesos de integración laboral de estos colectivos puesto que facilitan un empleo. En los Centros Especiales de Empleo el personal de formación, gestión y administración, desempeña un papel crucial como dinamizadores de la integración en el empleo de las personas con discapacidad. La percepción de este personal de la realidad y su modo de afrontarla en el trabajo diario, nos ofrece una nueva perspectiva apenas estudiada que permite un conocimiento más profundo de las empresas protegidas y, a su vez, facilita el conocimiento de las dificultades que las personas con discapacidad se encuentran en el desarrollo de sus procesos de integración sociolaboral.

La escasa trascendencia que se ha atribuido hasta la fecha a los profesionales empleados en las empresas protegidas y la inexistencia de estudios en Asturias sobre el papel crucial que desempeña el personal de formación, gestión y administración empleado en estos centros, como dinamizadores de la integración en el empleo de las personas con discapacidad, nos han llevado a realizar esta investigación.

\section{Objetivos}

De acuerdo con estos planteamientos, el objetivo fundamental de este trabajo es valorar las necesidades del Personal de Formación, Gestión y Administración empleado en los Centros Especiales de Empleo en Asturias.

\section{Fases del estudio}

Las fases de este estudio son las siguientes:

a) Análisis documental. Esta primera fase supone la búsqueda y recogida de documentación actual y relevante sobre los procesos de inserción en el mundo laboral de las personas con discapacidad

b) Analítica y descriptiva. Elaboramos cuestionarios semiestructurados y realizamos entrevistas abiertas. Una vez realizado el estudio estadístico de los datos cuantitativos y el análisis de la información cualitativa se describen detalladamente los resultados obtenidos sobre los cuales elaboramos las conclusiones de nuestro trabajo.

c) Evaluativa. En esta fase valoramos el proceso segundo, concretando los obstáculos que nos encontramos al realizar este estudio. Finalmente proponemos posibles medidas para tratar de resolver u optimizar los problemas identificados a lo largo de este trabajo. 


\section{Procedimientos de recogida de información}

Los instrumentos que utilizamos para recoger información necesaria para valorar las necesidades del personal de formación, gestión y administración empleado en los Centros Especiales de Empleo han sido cuestionarios semiestructurados y entrevistas abiertas.

Cuestionario: Valoración de necesidades del Personal de Formación, Gestión y Administración de los Centros Especiales de Empleo. Este cuestionario está compuesto por las 274 variables. Las variables de clasificación con las que hemos trabajado son cuatro: edad, sexo, tenencia o no de deficiencia y puesto que ocupa el encuestado en el Centro. Todas las variables se distribuyen en las cinco dimensiones que componen este cuestionario:

a) Datos sociodemográficos;

b) Objetivos y actividades del Centros Especial de Empleo;

c) Problemas en el trabajo diario y causas;

d) Percepción sobre el empleo de las personas con discapacidad;

e) Percepción sobre los factores favorecedores de la integración sociolaboral.

Entrevista abierta. Para completar la información obtenida a través de los cuestionarios decidimos también entrevistarnos con algunos representantes del personal de formación, gestión y administración. La entrevista responde a la necesidad de clarificar tres aspectos básicos de nuestro estudio:

1) los problemas que el personal de formación, gestión y administración se encuentra en su trabajo en los Centros Especiales de Empleo;

2) las causas de los mismos; y

3) las sugerencias de este personal para la mejora de la situación actual.

\section{Muestra}

La población de referencia la constituye la totalidad del personal de formación, gestión y administración empleado en los Centros Especiales de Empleo asturianos. La población que estimamos compone el personal de formación, gestión y administración, según datos de los 45 centros con los que contactamos, es de 150 personas (tamaño de la población $\mathrm{N}=150$ ). De este personal, participaron en nuestro estudio 62 personas (tamaño de la muestra $n=62$ ). Esta cifra constituye un $41 \%$ de la población, es decir más de un tercio del total de la población de referencia, por lo que la consideramos suficientemente representativa.

Tras la aplicación de los cuestionarios procedimos a seleccionar a las personas a entrevistar. De los 62 encuestados entrevistamos a 10 personas que desarrollan diversas funciones dentro del Centro: 3 son formadores, 3 son gerentes, 2 son administradores y 2 son gerentes que realizan también tareas administrativas. Este personal está empleado en cinco centros que por sus características y por el número de trabajadores con el que cuenta, consideramos que representan a los profesionales de los Centros Especiales de Empleo asturianos. 


\section{Técnicas de análisis de datos}

Análisis de la información obtenida con el cuestionario. Los datos cuantitativos recogidos con el cuestionario semiestructurado fueron analizados de modo descriptivo y relacional. Es decir, primero se hallaron las frecuencias válidas y acumuladas de los diversos ítems del cuestionario, que nos permitieron conocer el número de individuos u observaciones presentes en cada una de las categorías. Después se relacionaron todas las variables del cuestionario con las que ya en un primer momento habíamos seleccionado como variables de clasificación con las que íbamos a trabajar: la edad, el sexo, la tenencia o no de discapacidad y el tipo de puesto que la persona ocupaba en el centro.

Análisis de la información obtenida con las entrevistas. La información conseguida con las entrevistas ha sido cualitativa por lo que el tipo de análisis realizado es de contenido. La preguntas abiertas realizadas a los entrevistados nos han permitido recoger tanto los argumentos particulares expresados espontáneamente como los resultados del diálogo e interacción con la entrevistadora.

A partir de la información obtenida con los análisis descriptivos, relacionales y de contenido realizados, elaboramos las conclusiones de nuestro estudio.

\section{Principales conclusiones}

Las principales conclusiones obtenidas de la aplicación de los cuestionarios y la realización de las entrevistas al personal de formación, gestión y administración de los Centros Especiales de Empleo son las siguientes:

- La relativa novedad de la integración laboral de las personas con discapacidad conlleva un desconocimiento social que afecta al concepto que se tiene sobre los Centros Especiales de Empleo. Estos Centros funcionan como cualquier empresa, su $o b$ jetivo principal es tratar de integrar socialmente a las personas con discapacidad a través de su integración laboral. Entre los objetivos que estos centros no abordan en la realidad destaca el servir de puente a sus empleados hacia la empresa ordinaria, como la legislación señala, porque funcionan como cualquier empresa ordinaria. El único aspecto que diferencia a las empresas protegidas de las empresas ordinarias en el mercado laboral es el tipo de empleados, personas con discapacidad.

- La sociedad no cree en la capacidad productiva de las personas con discapacidad, y entienden este tipo de centros como una "obra social». La mayoría de los encuestados antes de trabajar en los Centros Especiales de Empleo estaban desempleados (42\%). Esta dificultad en sus trayectorias laborales se vincula a las deficiencias porque:

a) existe una mayor tasa de desempleo entre las personas con discapacidad; y

b) hay más experiencia en la empresa ordinaria entre personas sin discapacidad.

- La falta de personas con discapacidad cualificadas para responder a las demandas del mercado laboral es una de las causas principales que dificultan el acceso al empleo de estos colectivos. Entre las actividades que se realizan con menos frecuencia en los Centros Especiales de Empleo y que están consideradas por los encuestados como más importantes destacan las de formación continua de todo el personal. Los cambios realizados en el sistema educativo y las ofertas formativas de las entidades responsa- 
bles inadecuadas para estos colectivos, han contribuido a incrementar este problema. Por un lado, la formación que se ofrece a las personas con discapacidad no tiene en consideración su heterogeneidad, es decir, no diferencia los tipos de deficiencias que manifiestan. Por otro lado, faltan ofertas formativas que respondan a las necesidades de los profesionales que trabajan en contacto con personas con discapacidad

- El criterio principal que dificulta el acceso al trabajo en las empresas ordinarias de las personas con discapacidad es el tipo de deficiencia. Las personas con retraso mental van a ser las más perjudicadas debido a los falsos prejuicios y a los estereotipos sociales que se manifiestan hacia este colectivo vinculados al bajo rendimiento laboral y a la falta de hábitos, conductas y valores adecuados en relación al trabajo. El personal de formación, gestión y administración considera que la formación y las actitudes de los empresarios y de los trabajadores de la empresa ordinaria hacia el empleo de las personas con discapacidad, y en concreto de las personas con deficiencia mental, van a ser los factores principales que favorezcan la integración en el empleo no protegido de estos colectivos.

- El vacío legal que existe en lo referente a la jubilación de las personas con discapacidad obliga a los empresarios a adaptar el puesto de trabajo ante las nuevas limitaciones de la persona con deficiencias debidas a la edad o, a despedir al empleado. Para los entrevistados, este problema es debido a la falta de interés de los políticos en revisar y actualizar la legislación vigente sobre discapacidad, de modo que responda a las necesidades de estos colectivos.

- El personal de formación, gestión y administración señala como principales causas de estos problemas las siguientes:

1) la legislación por el concepto sobre Centros Especiales de Empleo que propone;

2) los políticos porque no cumplen sus promesas de empleo y mejora de la situación de las personas con discapacidad;

3) las organizaciones y entidades por ofrecer una formación desvinculada de las demandas del mercado laboral y de las necesidades concretas de cada uno de estos colectivos;

4) la sobreprotección familiar que limita la autonomía personal de las personas con dificultades; $y$

5) las personas con discapacidad por preferir el proteccionismo a su independencia.

- Las medidas que este personal considera oportunas para mejorar la situación de la integración laboral de los empleados con discapacidad en las empresas protegidas son las siguientes:

a) revisar la legislación vigente;

b) crear una asociación a nivel estatal y autonómico de Centros Especiales de Empleo;

c) promover la difusión social de la imagen y la idea de estos Centros como empresas; $y$

d) adoptar una política de formación en Asturias que responda a las necesidades de las personas con discapacidad en general, y en particular del personal de formación, gestión y administración y el personal empleado de los Centros Especiales de Empleo. 


\section{Propuestas de mejora}

A continuación proponemos las actuaciones que nos parecen necesarias para lograr una verdadera integración sociolaboral de las personas con discapacidad:

1. Actualizar la legislación vigente en materia de discapacidad y llevar a cabo políticas sociales que impulsen la integración de estos colectivos.

2. Adecuar las acciones de la Administración Pública a las necesidades reales de los Centros Especiales de Empleo.

3. Potenciar la formación de las personas con discapacidad como principal recurso de estos colectivos para su inserción laboral.

4. Potenciar la formación permanente de los profesionales que trabajan en contacto con personas con discapacidad.

5. Concienciar a las familias de que una educación sobreprotectora limita las posibilidades de estas personas.

6. Sensibilizar a la sociedad de las posibilidades de este colectivo.

7. Incrementar el apoyo y financiación de investigaciones vinculadas a las necesidades de formación y empleo de las personas con discapacidad.

\section{Referencias bibliográficas}

De Miguel, M., Pereira, M. y col. (1999). Análisis de las necesidades y problemas en la población con deficiencias auditivas en el proceso de integración en el mundo laboral. La realidad en Asturias. Oviedo: Universidad de Oviedo/MEC.

Fondo-Formación (1998). El formador ante la discapacidad. Manual para profesionales. Avilés: Servicio de Publicaciones del FPE-Fondo Formación.

Garrido Pérez, E. (2000). El trabajo de los minusválidos en Centros Especiales de Empleo. Valencia: Tirant Lo Blanch.

Martínez Rueda, N. (1994). Una experiencia de formación de monitores de talleres protegidos en la Comunidad Autónoma Vasca, en Ortiz González, M.C. (Coord.): Integración sociolaboral de las personas con minusvalía. Actas del Seminario Europeo EASE-AEDES. Salamanca 1992. Madrid: MAPFRE, 115-124.

Ministerio de Trabajo y Asuntos Sociales (2000). Guía laboral 2000 y de asuntos sociales. Madrid: Ministerio de Trabajo y Asuntos Sociales.

Pereda, C., De Prada, M. A., Actis, W., Santamarina, C., Camas, V. (1998). Discapacidad y trabajo en España. Estudio de los procesos de inclusión y exclusión social de las personas con discapacidad. Madrid: Ministerio de Trabajo y Asuntos Sociales.

Sánchez Cervera y Senra, J. M. (2000). Los trabajadores discapacitados: contratación, incentivos, centros especiales de empleo, prestaciones sociales, fiscalidad. Madrid: CISS.

Tuset Del Pino, P. (2000). La contratación de trabajadores minusválidos. Pamplona: Aranzadi. 